\title{
Selection of Remote Phosphor Led Packages FOR IMPROVING LUMINOUS FLUX
}

\author{
Ming Jui CHEN ${ }^{1}$, Hsiao-Yi LEE ${ }^{1}$, Doan Quoc Anh NGUYEN ${ }^{2, *}$, \\ Thi Phuong Loan NGUYEN ${ }^{3}$, Van Tho LE $E^{4}$
}

\author{
${ }^{1}$ Department of Electrical Engineering, National Kaohsiung University of Science and \\ Technology, Kaohsiung, Taiwan \\ ${ }^{2}$ Power System Optimization Research Group, Faculty of Electrical and Electronics Engineering, \\ Ton Duc Thang University, Ho Chi Minh City, Vietnam \\ ${ }^{3}$ Faculty of Fundamental 2, Posts and Telecommunications Institute of Technology, \\ Ho Chi Minh City, Vietnam \\ ${ }^{4}$ Institute of Tropical Biology, Vietnam Academy of Science and Technology, \\ Ho Chi Minh City, Vietnam
}

Corresponding Author: Doan Quoc Anh NGUYEN (Email: nguyendoanquocanh@tdtu.edu.vn)

(Received: 19-Sep-2019; accepted: 03-Feb-2020; published: 30-Jun-2020)

DOI: http://dx.doi.org/10.25073/jaec.202042.259

\begin{abstract}
In this paper, the first issue presented and analyzed by several experiments is the influence of the distance between phosphor layers in the dual-layer and triple-layer remote package on luminous flux and color rendering property. During the simulation, it was realized that it was possible to create a suitable distance to create higher quality white light-emitting diodes (WLEDs) by adjusting the distance between the phosphor layers. According to the study results, $0.1 \mathrm{~mm}$ is the most reasonable distance between two phosphor layers so that the performance of the multi-chip white light LED (MCW LED) can get the best optimal effect. Through a series of experiments, it has been proved that the efficiency of the two-layer structure gives optical properties higher than the three-layer structure related to distance. The highest achievable lumen output is $0.6 \mathrm{~mm}$ for triple-layer structure and $0.1 \mathrm{~mm}$ for dual-layer structure. Meanwhile, the color rendering index changes insignificantly when the distance increases. The triplelayer package is not practical for high-power white LEDs due to the high cost and low conversion efficiency. The dual-layer remote phosphor package with a $0.1 \mathrm{~mm}$ phosphorus gap is the op-
\end{abstract}

timal structure of WLED in improving luminous efficiency and color rendering index.

\section{Keywords}

Multi-chip white LEDs, remote phosphor package, luminous efficacy, color rendering index.

\section{Introduction}

Due to the quick development in digital illumination market, traditional lighting sources become completely obsolete, which is the reason why phosphor-converted white light emitting diodes (pc-LEDs) tend to become a promising lighting source with many opportunities to compete with the traditional ones in several applications [1-3]. However, available pc-LEDs still confront some imperfections that need to be improved and optimized, such as low conversion efficiency and color rendering ability, and poor 
color uniformity. Currently, the yellow emitting phosphor of white LED structures is directly applied onto the LED chip surface, which causes the thermal degradation of phosphor material, resulting in the reduction of reliability of products [4-6]. Narendran with his partners demonstrated that more than half the downconverted light is backscattered and reflected inside the LED, leading to a reduction of the overall light output [7]. To limit the rays-trapped phenomenon inside the LED, a new technology called remote phosphor package is discovered. In this structure, the phosphor layer is placed at a sufficiently large distance from the LED chip, thereby detecting the backscattered photons and improving the luminous efficacy.

Due to the existence of an air gap between the LED and the phosphor layer, the effect of heat can be reduced during the optical performance, which leads to more blue and yellow rays being converted and transmitted toward the LED surface than the conventional cases and yielding better optical properties. In development, the next generation of remote phosphor configuration is proposed, in which the dual-layer structure employs the remote phosphor package to enhance the luminous efficiency of pc-LEDs [8]. This study indicated that if dual-layer remote phosphor structure uses a thin silicon layer, it will get $5 \%$ better lumen output than a conventional one at the same CCT. Similarly, several methods were included in the study in order to lessen the power of the light backward from the phosphor layer to the absorptive LED chip. There are typically three distinct types of phosphor structure that are ring remote phosphor structure, remote phosphor package with the hemispherical dome, and remote phosphor package employing a double remote micro-patterned phosphor film into pc-LEDs [9-14]. Moreover, another proposition also demonstrates that an air gap layer embedded in a remote phosphorus package has the advantage of luminous flux and color stability [15-18]. Light transmitted back to the LED chip of air-gap embedded white LED is less than that of the conventional one, and thus, the lumen output was enhanced. In addition, the difference in phosphor material arrangement plays a significant role in improving phosphorus conversion efficiency. A double-layer remote phosphor structure with a $\mathrm{SrO} .3 \mathrm{~B}_{2} \mathrm{O}_{3}: \mathrm{Sm}^{2+}$ red phosphor layer above a YAG:Ce ${ }^{3+}$ yellow phosphor layer can yield $17 \%$ more lumen than the mixed red and yellow phosphor package [19]. However, these phosphor layers in this structure are placed adjacent to each other at a random distance with the LED chip, and the specified distance between the LED chip with phosphor layers has not been determined in previous studies. That's why this paper has an aim to propose the most appropriate distance from LED chip to phosphor layers for the greatest optical performance.

This article has compared the difference in the effect of distance between phosphor layers and LED chips of two-layer and three-layer structures on optical properties, as shown in Figure 1, starting with luminous efficiency and color rendering ability. In order to achieve an optimized design for developing the high-efficiency LED, the right position of phosphor layers within the LED package must have been determined. The effect enhancement is due to the improvement in the use of blue and yellow rays in LEDs by adjusting the distance between phosphor layers and the LED chip. The simulation results pointed out that the LEDs of the two-layer remote phosphor package exhibits the ability to lighten and display color better than triple-layer one.

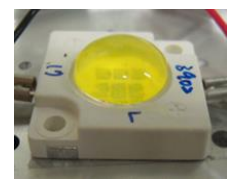

(a)

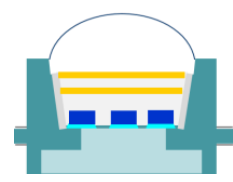

(b)

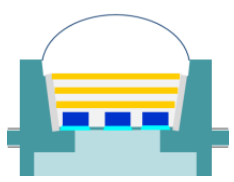

(c)
Fig. 1: (a) Experimented LED package, (b) Dual-layer phosphor structure, and (c) Triple-layer phosphor structure.

\section{Simulation and computation}

The effect of the distance between phosphor layers with an LED chip on the light output of pcLED was examined using 3 -D ray detection simulation with LightTools software. A thin phos- 
phor layer of remote phosphor package is separated far away from the LED chip by a transparent material layer. The simulation about the effect of distance between two phosphor layers for two different configuration packages on the optical characteristics of LED is performed by the 3-D model. Each blue LED chip has a 1.16 $\mathrm{W}$ radiation flux at $455 \mathrm{~nm}$ emission wavelength with a height of $0.15 \mathrm{~nm}$ and a $1.14 \mathrm{~nm}$ square base within the cavity of the reflector. 1.8 is a uniform refractive index for phosphor particles at all wavelengths of light emitted. While the shape of the phosphor particle is spherical and the average diameter is $14.5 \mathrm{~nm}$, the reflector has a height of $2.07 \mathrm{~nm}$ and a bottom length of $8 \mathrm{~nm}$. Each package has a convex lens boned on the top surface of the phosphor layer. The refractive index of the material for the convex lens is 1.46 at the excitation wavelength of $460 \mathrm{~nm}$. Since the CCT value of simulated WLED is 8500 $\mathrm{K}$, the concentration of phosphor in both duallayer and tri-layer package is constantly changing to maintain the same CCT of white LEDs during the simulation process as shown in Figure 2. Also in Figure 2, the concentration in the dual-layer package has an upward trend with the distance from 0 to $0.1 \mathrm{~mm}$ and this value stays the same afterward. Meanwhile, the concentration of the tri-layer package rapidly increases in the distance ranging from $0.2-0.4 \mathrm{~mm}$ and slightly decreases when it continuously increases to $0.7 \mathrm{~mm}$. The concentration and the thickness of phosphor layers for two cases of structures are set the same.

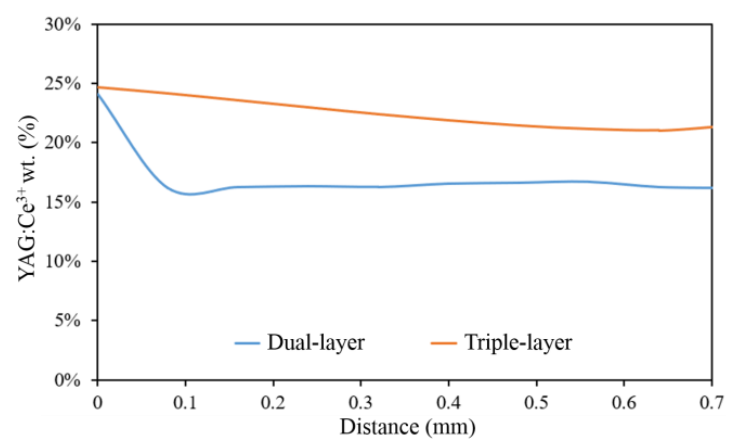

Fig. 2: The concentration of YAG: $\mathrm{Ce}^{3+}$ phosphor with different distances between two phosphor structures at the same CCT of white LED.
For the transmitted blue light and blue light at the boundaries of the first layer

$$
\begin{aligned}
P B_{1} & =P B_{0} e^{-2 \alpha B_{1} h} \\
P Y_{1} & =\frac{1}{2} \frac{P B_{0}}{\alpha B_{1}-\alpha Y_{1}}\left(e^{-2 \alpha Y_{1} h}-e^{-2 \alpha B_{1} h}\right)
\end{aligned}
$$

For the transmitted blue light and blue light at the boundaries of the second layer

$$
\begin{aligned}
P B_{2}= & P B_{0} e^{-\alpha B_{2} h} e^{-\alpha B_{2} h}=P B_{0} e^{-2 \alpha B_{2} h} \\
P Y_{2}= & \frac{1}{2} \frac{\beta_{2} \times P B_{0}}{\alpha B_{2}-\alpha Y_{2}}\left(e^{-2 \alpha Y_{2} h}-e^{-2 \alpha B_{2} h}\right) \\
& \times e^{-2 \alpha Y_{2} h}+\frac{1}{2} \frac{\beta_{2} P B_{0} e^{-\alpha B_{2} h}}{\alpha B_{2}-\alpha Y_{2}} \\
& \times\left(e^{-\alpha Y_{2} h}-e^{-\alpha B_{2} h}\right) \\
= & \frac{1}{2} \frac{\beta_{2} P B_{0} e^{-\alpha B_{2} h}}{\alpha B_{2}-\alpha Y_{2}}\left(e^{-2 \alpha Y_{2} h}-e^{-2 \alpha B_{2} h}\right)
\end{aligned}
$$

where $h$ is the notation of the thickness of each layer, while the subscript " 1 " and "2" are used to describe the first and the second phosphor layer, $\beta$ represents the conversion coefficient for blue light converting to yellow light, $\gamma$ is reflection coefficient, $\rho_{v}$ is the particle density; $P B, P Y$ are the intensities of blue light and yellow light respectively. $\alpha, \beta$ are parameters describing the fractions of the energy loss of blue and yellow lights.

In addition, the paper demonstrated that the lighting efficiency pc-LEDs in the double-layer phosphor structure enhances:

$$
\frac{\left(P B_{2}+Y P_{2}\right)-\left(P B_{1}+P Y_{1}\right)}{P B_{1}+P Y_{1}}>0
$$

The simulation result and the theoretical calculation verify that the transmitted light increases and the backscattering light (by the phosphor layer) degrades in the structure of double-layer package, which improves the lightextraction efficiency of white LED.

By using the Mie theory [20, 21], the scattering of phosphor particles was analyzed. Additionally, in the following expression by using the Mie theory, the scattering cross section $\mathrm{C}_{\text {sca }}$ for spherical particles can be calculated. The 
transmitted light power can be computed by the Lambert-Beer law:

$$
I=I_{0} \exp \left(-\mu_{\text {ext }} L\right)
$$

In this formula, $I_{0}$ is the incident light power, $L$ is the phosphor layer thickness $(\mathrm{mm})$, and $\mu_{\text {ext }}$ is the extinction coefficient, which can be expressed as: $\mu_{\text {ext }}=N_{r} . C_{\text {ext }} . \quad N_{r}$ is the number density distribution of particles $\left(\mathrm{mm}^{-3}\right)$ and $C_{\text {ext }}\left(\mathrm{mm}^{2}\right)$ is the extinction cross-section of phosphor particles.

\section{Results and discussion}

To enhance light efficiency and LED color quality, as well as benefit the thermal management of remote phosphor, a remote phosphor simulation model was developed. This model allows adjusting the phosphor position to find the optimal distance between the phosphor layers and LED chips to build the optical properties of the LED. During the simulation, the position of the middle phosphorus layers must be adjusted while the top phosphorus layer should be fixed. However, the reflection, absorption, scattering, conversion, and transmission of light in LED can lead to the variation of the correlated color temperature of LED according to the location and arrangement of the phosphor layers in both duallayer and tri-layer package. Therefore, the phosphor concentration needs to change with the distance between the phosphor and LED chips, as shown in Figure 2, to be able to preserve the CCT of this package.

The phosphor concentration of dual-layer package tends to decrease in the range $0-0.1$ $\mathrm{mm}$ and does not change afterward. It can be assumed that two phosphor layers placed at a short distance of $0-0.1 \mathrm{~mm}$ looks like a single phosphor layer with a double thickness. As known in the previous studies, increasing the phosphor layer thickness can enhance the scattering, the absorption, and the reflection, thus causing a high color correlation temperature within the LED package. Therefore, it is necessary to reduce the phosphor concentration to ensure that this package has the same CCT during the simulation. The YAG: $\mathrm{Ce}^{3+}$ phosphor concentration remains the same when the distance increases by more than $0.1 \mathrm{~mm}$ in two-layer structure and significantly increases by more than $25 \%$ when the distance is about $0.2-0.4 \mathrm{~mm}$ in tri-layer structure. When the distance $\mathrm{d}$ varies from $0.1-0.7$ $\mathrm{mm}$, the concentration varies slightly.

Figure 3 shows the influence of the distance between phosphor layers and LED chip of remote phosphor package on the lumen output. The result pointed out that light extraction depends on the variation of the distance. For the dual-layer package, the lumen output enhances significantly and reaches the highest value in the distance of phosphor layers of $0.5-0.6 \mathrm{~mm}$. In contrast, when the distance increases more than $0.6 \mathrm{~mm}$, the luminous flux tends to decrease. It seems that, as the distance $d$ changes from 0.5 to $0.6 \mathrm{~mm}$, the two phosphor layers are further separated and the probability of light rays trapped in the distance between the two layers of phosphorus will decrease. The reflected light shining directly onto the absorption LED chip can be reduced and the light passing through the phosphorus layers can be increased, thus improving the efficiency of the LED. With a distance of $0.5-0.6 \mathrm{~mm}$, heat generated by the LED chip only transfers to the substrate instead of the contact surface of two phosphor layers. The distance growth of phosphor layers may not only improve luminous flux but also create temperature stability of this multi-layer phosphor configuration, which results in more light being transmitted and extracted through phosphorus layers. For a range of $0.6 \mathrm{~mm}$ to $0.7 \mathrm{~mm}$ distance, the reduction of luminous flux is attributed to the weak photon extraction and the thermal effect of phosphor. Blue light from LED chip will encounter the first phosphor layer and convert the blue light to the yellow light. However, part of the light is lost inside the LED due to backscattering, absorption, and reflection, the rest is turned into yellow light and transmitted through the second phosphorus layer. The increasing distance makes the phosphorus layer move closer to the LED chip, and therefore more light is trapped inside the gap between this phosphorus layer and the LED chip, as well as the back-reflected light to the LED chip. This causes an increase in the junction temperature of both phosphorus and LED chips, which can produce 
low conversion efficiency. For the three-layer structure, the process of propagating light inside the LED has an opposite tendency. The luminous flux also increases at the beginning of the distance of $0-0.1 \mathrm{~mm}$ and decreases at the position of $0.1-0.7 \mathrm{~mm}$ distance. The simulation results show that the case of the doublelayer package has better improvement than the three-layer package as shown in Figure 3. PcLEDs with the dual-layer remote phosphor package show a rapid increase of the luminous flux which is higher than that of the triple-layer phosphor package at the beginning. The photons are extracted into the total light energy of the two-layer configuration including higher content than the three-layer configuration.

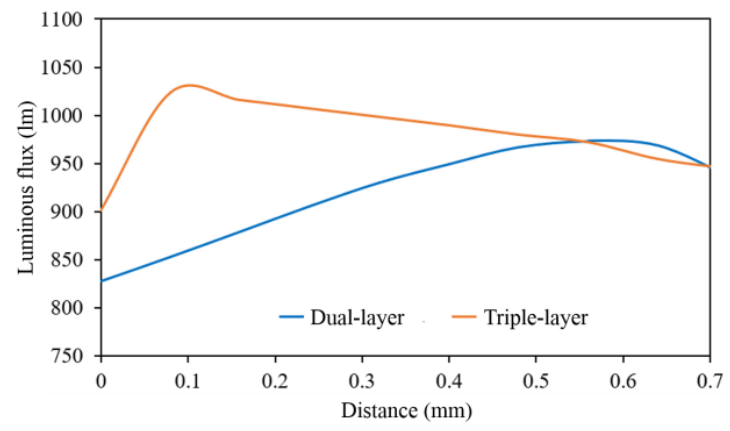

Fig. 3: The luminous efficiency of white LEDs at the same CCT with different distances between two phosphor structures.

In addition, the effect of the distance between two layers of phosphorus on the color rendering index was studied. For both types of multi-layer configurations, when the distance is appropriate, the probability of the transition intensity and the transmission intensity of blue light and yellow light increase. The heat generated by LED chips often transfers into the substrate instead of phosphor, which helps avoid the chemical change of phosphor as well as the destruction of LEDs due to heat problems. This provides a wider emission spectrum at this location of the phosphorus layer and makes the color quality of the LED and the CRI better at the same time. When the distance is too large or the first phosphorus layer is very close to the LED chip, the blue rays are scattered back and trapped inside the LED package. Consequently, the light quality becomes worse and hence the color rendering index is low. Although the CRI trend for both cases of multi-layer phosphorus configuration is the same, it can be observed that the CRI of the dual-layer phosphor configuration is better than that of the tri-layer one as shown in Figure 4. In conclusion, the change in position, as well as the amount of phosphorus in the remote phosphorus package, significantly affected the luminous flux, CRI. The best luminous flux and CRI can be achieved at the optimal distance of $0.1 \mathrm{~mm}$ in a two-layer remote phosphorus package. The twolayer package offers great advantages in bringing high luminous flux and CRI. The blue light and yellow light must pass through three layers of phosphorus in the three-layer package, so there is still a large possibility that a ray of light is lost inside these three layers of phosphorus.

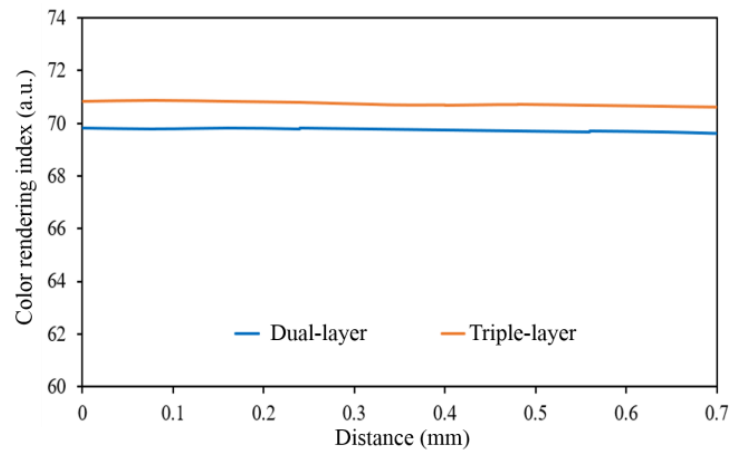

Fig. 4: The color rendering index (CRI) value of white LEDs with different distance between two phosphor layers at the same CCT.

\section{Conclusion}

In this study, several experiments were carried out in order to analyze and demonstrate the influence of the distance between phosphor layers and LED chip as well as the number phosphor layers on the pc-LED optical characteristics at the same CCT. The suitable position of phosphor layer in remote phosphor package which is discovered via several experiments can have the luminous flux and color rendering index of LED significantly bettered. In addition, the luminous flux remarkably enhances and reaches the maximum value at the distance of $0.6 \mathrm{~mm}$ for the case of trial-layer package and $0.1 \mathrm{~mm}$ for duallayer package. Meanwhile, with the distance ranging from $0.1 \mathrm{~mm}$ to $0.7 \mathrm{~mm}$ of dual-layer 
package, the lumen output and the color quality of LEDs have a slight downward tendency. The increase of the trapping, the absorption, the re-scattering of light in LED package, and the chemical change of the heated phosphor layer are the main reason for this issue. Therefore, the key issue of this scientific paper is how to find an appropriate space between phosphor layers in remote phosphor package in designing pc-LEDs with outstanding efficiency.

\section{References}

[1] Anh, N. D. Q., Le, P. X., \& Lee, H. Y. (2019). Enhanced luminous flux of white LED using flat dual-layer remote phosphor configuration. Journal of Advanced Engineering and Computation, 3(2), 426-432.

[2] Li, C. N., Rao, H. B. Zhang, W. Zhou, C. Y. Zhang, Q., \& Zhang, K. (2016). Self-Adaptive Conformal-Remote Phosphor Coating of Phosphor-Converted White Light Emitting Diodes. Journal of Display Technology, 12(9), 946-950.

[3] Anh, N. D. Q, Le, P. X., \& Lee, H. Y. (2019). Selection of a Remote Phosphor Configuration to Enhance the Color Quality of White LEDs. Current Optics and Photonics, 3(1), 78-85.

[4] Li, Z. T., Wang, H. Y. Yu, B. H. Ding, X. R., \& Tang, Y. (2017). High-efficiency LED COB device combined diced $\mathrm{V}$-shaped pattern and remote phosphor. Chinese Optics Letters, 15(4).

[5] Ying, S. P., \& Shen, J. Y. (2016). Concentric ring phosphor geometry on the luminous efficiency of white-light-emitting diodes with excellent color rendering property. Optics Letters, 41(9), 1989-1992.

[6] Chiang, C. H., Tsai, H. Y. Zhan, T. S. Lin, H. Y. Fang, Y. C., \& Chu, S. Y. (2015). Effects of phosphor distribution and stepindex remote configuration on the performance of white light-emitting diodes. Optics Letters, 40(12), 2830-2833.
[7] Yu, S. D., Li, Z. T. Liang, G. W. Tang, Y. Yu, B. H., \& Chen, K. H. (2016). Angular color uniformity enhancement of white light-emitting diodes by remote micropatterned phosphor film. Photonics Research, 4(4), 140-145.

[8] Jeon, S. W., Kim, S. H. Choi, J. N. Jang, I. S. Song, Y. H. Kim, W. H., \& Kim, J. P. (2018). Optical design of dental light using a remote phosphor light-emitting diode package for improving illumination uniformity. Applied Optics, 57(21), 5998-6003.

[9] Xie, B., Chen, W. Hao, J. N. Wu, D. Yu, X. J. Chen, Y. H. Hu, R. Wang, K., \& Luo, X. B. (2016). Structural optimization for remote white light-emitting diodes with quantum dots and phosphor: packaging sequence matters. Optics Express, 24(26), A1560-A1570.

[10] Li, J. S., Li, Z. T. Liang, G. W. Yu, S. D. Tang, Y., \& Ding, X. R. (2016). Color uniformity enhancement for COB WLEDs using a remote phosphor film with two freeform surfaces. Optics Express, 24(21), 23685-23696.

[11] Shih, B. J., Chiou, S. C. Hsieh, Y. H. Sun, C. C. Yang, T. H. Chen, S. Y., \& Chung, T. Y. (2015). Study of temperature distributions in pc-WLEDs with different phosphor packages. Optics Express, 23(26), 3386133869 .

[12] Guo, Z. Q., Lu, H. L. Shih, T. M. Lin, Y. Lu, Y. J., \& Chen, Z. (2016). Spectral Optimization of Candle-Like White LightEmitting Diodes With High Color Rendering Index and Luminous Efficacy. Journal of Display Technology, 12(11), 1393-1397.

[13] Ma, R., Ma, C. Y., Zhang, J. T., Long, J. Q., Wen, Z. C., Yuan, X. Y., \& Cao, Y. G. (2017). Energy transfer properties and enhanced color rendering index of chromaticity tunable green-yellow-red-emitting $\mathrm{Y}_{3} \mathrm{Al}_{5} \mathrm{O}_{12}: \mathrm{Ce}^{3+}, \mathrm{Cr}^{3+}$ phosphors for white light-emitting diodes. Optical Materials Express. $7(2), 454-467$.

[14] Anh, N. D. Q., Vinh, N. H., \& Lee, H. Y. (2017). Effect of Red-emitting 
$\mathrm{Sr}_{2.41} \mathrm{~F}_{2.59} \mathrm{~B}_{20.03} \mathrm{O}_{74.8}: \mathrm{Eu}_{0.12}, \mathrm{Sm}_{0.048}$ Phosphor on Color Rendering Index and Luminous Efficacy of White LEDs. Current Optics and Photonics, 1(2), 118-124.

[15] Li, N. Q., Zhang, Y. Quan, Y. W. Li, L. Ye, S. H. Fan, Q. L., \& Huang, W. (2018). Highefficiency solution-processed WOLEDs with very high color rendering index based on a macrospirocyclic oligomer matrix host. Optical Materials Express, 8(10), 3208-3219.

[16] Li, Y. Y., Chen, J. J. Feng, H. H. Chen, H., \& Wang, Q. M. (2014). Rugate filters used in slit-lamp delivery to improve color rendering of illumination for retinal photocoagulation. Applied Optics, 53(15), 33613369 .

[17] Ou, Y. Y., Corell, D. D., Dam-Hansen, C. Petersen, P. M., \& Ou, H. Y. (2011). Antireflective sub-wavelength structures for improvement of the extraction efficiency and color rendering index of monolithic white light-emitting diode. Optics Express, 19(S2), A166-A172.

[18] He, G. X., \& Tang, J. (2015). Study on the correlations between color rendering indices and the spectral power distributions: comment. Optics Express, 23(3), A140-A145.

[19] Hung, P. C., \& Tsao, J. Y. (2013). Maximum White Luminous Efficacy of Radiation Versus Color Rendering Index and Color Temperature: Exact Results and a Useful Analytic Expression. Journal of Display Technology, 9(6), 405-412.

[20] Huyal, I. O., Ozel, T. Koidemir, U. Nizamoglu, S. Tuncel, D., \& Demir, V, H. (2008). White emitting polyfluorene functionalized with azide hybridized on nearUV light emitting diode for high color rendering index. Optics Express, 16(2), 11151124 .
[21] Lee, H. Y., Le, P. X., \& Anh, N. D. Q. (2019). The Impacts of Red-emitting $\mathrm{Mg}_{2} \mathrm{TiO}_{4}: \mathrm{Mn}^{4+}$ Phosphor on Color Quality of Dual-layer Remote Phosphor Configuration. Journal of Advanced Engineering and Computation, 3(3), 464-470.

\section{About Authors}

Ming Jui CHEN was born in Tainan city, Taiwan. He has been working at the Department of Electrical Engineering, National Kaohsiung University of Science and Technology, Kaohsiung, Taiwan. His research interest is optical material.

Hsiao-Yi LEE was born in Hsinchu city, Taiwan. He has been working at the Department of Electrical Engineering, National Kaohsiung University of Science and Technology, Kaohsiung, Taiwan. His research interest is optics science.

Doan Quoc Anh NGUYEN was born in Khanh Hoa province, Vietnam. He has been working at the Faculty of Electrical and Electronics Engineering, Ton Duc Thang University. Quoc Anh received his PhD degree from National Kaohsiung University of Science and Technology, Taiwan in 2014. His research interest is optoelectronics.

Thi Phuong Loan NGUYEN was born in Da Nang province. In 2006, She received her master degree from University of Natural Sciences. Her research interest is optoelectronics. She has worked at the Faculty of Fundamental 2, Posts and Telecommunications Institute of Technology, Ho Chi Minh City, Vietnam.

Van Tho LE was born in Thanh Hoa province, Vietnam. In 2019, he received his master degree from the University of Science - Vietnam National University. His research interest is optoelectronics. He has worked at Institute of Tropical Biology, Vietnam Academy of Science and Technology. 